\title{
Symmetric reduction of high-multiplicity one-loop integrals and maximal cuts
}

\section{Guy R. Jehu}

School of Mathematics and Hamilton Mathematical Institute, Trinity College Dublin, College Green, Dublin, Ireland

E-mail: jehu@maths.tcd.ie

ABSTRACT: We derive useful reduction formulae which express one-loop Feynman integrals with a large number of external momenta in terms of lower-point integrals carrying easily derivable kinematic coefficients which are symmetric in the external momenta. These formulae apply for integrals with at least two more external legs than the dimension of the external momenta, and are presented in terms of two possible bases: one composed of a subset of descendant integrals with one fewer external legs, the other composed of the complete set of minimally-descendant integrals with just one more leg than the dimension of external momenta. In 3+1 dimensions, particularly compact representations of kinematic invariants can be computed, which easily lend themselves to spinor-helicity or trace representations. The reduction formulae have a close relationship with $D$-dimensional unitarity cuts, and thus provide a path towards computing full (all- $\epsilon$ ) expressions for scattering amplitudes at arbitrary multiplicity.

KEYwords: Scattering Amplitudes, Perturbative QCD, Models of Quantum Gravity, Supersymmetric Gauge Theory

ArXiv EPrint: 2010.16266 


\section{Contents}

1 Introduction 1

2 Review and definitions 3

3 Four-dimensional Minkowski space 5

3.1 Pentagon 5

3.2 Hexagon 6

4 Reduction formulae $\quad 8$

4.1 Generalised reduction of scalar integrals 8

$\begin{array}{lll}4.2 & \text { Feynman parameters in the numerator } & 10\end{array}$

5 Maximal cuts $\quad 12$

5.1 The $D$-dimensional pole 13

6 Concluding remarks $\quad 15$

$\begin{array}{ll}\text { A Explicit derivations } & 16\end{array}$

A.1 $n=L+3$ reduction 16

\section{Introduction}

The computation of multi-loop helicity amplitudes in Yang-Mills theories has recently met with great success, such as at two loops, five points for QCD [1-5], and, using myriad mathematical tools, through to seven loops and six points for planar $\mathcal{N}=4$ super YangMills [6]. Despite this, all multiplicity (all- $n$ ) analytic forms of one-loop amplitudes in YangMills theory are known only up to $\mathcal{O}(\epsilon)$, and only in cases with maximal supersymmetry and MHV helicity structure [7], or all-plus and single-minus helicity configurations [8, 9]. Recently, an all- $n$ form for a two-loop partial all-plus amplitude in pure Yang-Mills has been computed [10]. One of the principle barriers to computing these is a lack of a concise analytic understanding of one-loop high-multiplicity integrals.

One way this is relevant to contemporary multi-loop computations is that all- $\epsilon$ analytic expressions occur when applying one-loop results to multi-loop integrals [11, 12]; recent computations of all-plus amplitudes at two loops reveal a tantalisingly one-loop like analytic structure [4,13-16]. Moreover, higher-in-epsilon terms of one-loop amplitudes contribute at two-loops Such an amplitude $A_{n}^{2-\text { loop }}$ can be arranged in a way that manifests its universal infra-red (IR) structure [17]:

$$
A_{n}^{2-\text { loop }}=A_{n}^{\text {tree } \mathfrak{I}^{2}}+A_{n}^{1-\text { loop } \mathfrak{I}^{1}+F_{n},}
$$


where the universal infra-red terms $\mathfrak{I}^{m}$ are defined in [17]. In particular, the term $\mathfrak{I}^{1}$ contains infra-red poles in the dimensional regulator up to $\mathcal{O}\left(\epsilon^{-2}\right)$ which would cancel with the higher-in- $\epsilon$ terms in the one-loop amplitude $A^{1-\text { loop }}$ upon expansion, leaving subleading and extra finite terms in addition to $F_{n}$.

Beyond this, the all- $\epsilon$ one-loop structure of $\mathcal{N}=4$ super Yang-Mills is conjectured to be proportional to a dimension-shifted all-plus amplitude in pure Yang-Mills [18]; a conclusive proof of this is still outstanding, but will be addressed in a forthcoming paper [19]. Generalising results such as these which rely on particular contingencies requires a more complete understanding of the all- $n$ analytic structure of both tree amplitudes and integrals; this paper focuses on one-loop instances of the latter.

Techniques to reduce finite $n$-point one-loop Feynman integrals (from here on referred to as an $n$-gon) in integer dimension have long been well understood [20-24]. The use of dimensional regularisation to deal with both ultraviolet (UV) and infra-red (IR) divergencies led to more general formulae $[25,26]$ which both justify a small basis of scalar integrals for one-loop amplitudes and codify relations amongst the integrals as a particular case of dimensional recurrence identities [27]. These can in turn be used to solve the integrals themselves [27-31].

These formulae face issues, however, when $n>2+L$, for $L$ the dimension of the external momenta, ${ }^{1}$ in which case the derivation depends on the inversion of a singular matrix $S$. A formalism was in turn developed [33] in terms of defining a pseudo-inverse of $S$ to resolve high- $n$ integrals into lower-point ones. Although suitable for application in the form of computer algorithms, the approach breaks the cyclic symmetry of the external legs, obscuring the simplicity of the analytic structure and making it difficult to apply to possible conjectures of all- $n$ all- $\epsilon$ forms.

In this paper we present an alternative which preserves the symmetries when reduced to $(L+1)$-gons, and allows simple basis choices to be made for the reduction to $(n-1)$ gons. For the equal propagator-mass case where $L=4$, the reduction formula from $n$-point integrals $^{2} I_{n}$ to pentagon integrals $I_{5}$ is especially simple and takes the form

$$
\begin{aligned}
I_{n} & =\frac{1}{2^{n-5}} \sum_{i_{1}, \ldots, i_{n-5}=1}^{n}\left[\prod_{m}^{n-5} \xi_{i_{m}}^{\left[\mathcal{P}_{i}, i_{m}\right]}\right] I_{5}^{\left[\mathcal{P}_{i}\right]}, \\
\mathcal{P}_{i} & =\{1, \ldots, n\} /\left\{i_{1}, \ldots, i_{n-5}\right\}
\end{aligned}
$$

where

$$
\xi_{k_{j}}^{\left[k_{1}, k_{2}, k_{3}, k_{4}, k_{5}, k_{6}\right]}=(-1)^{j} \frac{2 \operatorname{tr}_{5}\left(q_{k_{j+1} k_{j+2}} q_{k_{j+2} k_{j+3}} q_{k_{j+3} k_{j+4}} q_{k_{j+4} k_{j+5}}\right)}{\operatorname{tr}_{5}\left(q_{k_{1} k_{2}} q_{k_{2} k_{3}} q_{k_{3} k_{4}} q_{k_{4} k_{5}} q_{k_{5} k_{6}} q_{k_{6} k_{1}}\right)} ;
$$

here $\operatorname{tr}_{5}\left(p_{a} p_{b} \ldots\right)=\operatorname{tr}\left(\gamma_{5} \not p_{a} \not p_{b} \ldots\right), q_{i j}=p_{i}+p_{i+1}+\ldots+p_{j-1}$ where $p_{i}$ is the (cyclically indexed) $i$ th outgoing external momentum.

The paper is structured as follows: in section 2 the notation is introduced, and we review derivations of some previously known results. In section 3 we explicitly study

\footnotetext{
${ }^{1}$ We assume that the external legs are always kept in integer dimension, for example in the fourdimensional helicity scheme of dimensional regularisation [32].

${ }^{2}$ Normalisations and definitions are found in section 2.
} 


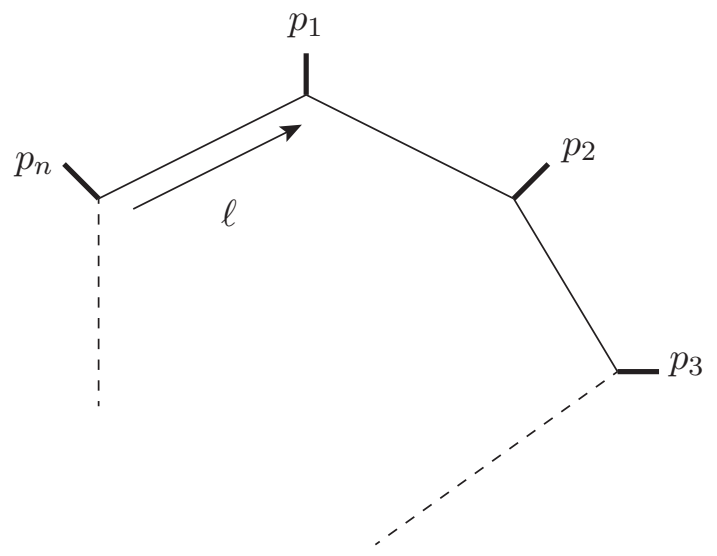

Figure 1. Diagrammatic representation of the general one-loop integral function $\mathcal{I}_{n}^{D}$ in eq. (2.1). The external momenta $p_{i}$ should always be considered outgoing.

the case where the external dimensions are in four-dimensional Minkowski space, giving explicit analytic expressions for the various kinematic determinants and coefficients for the pentagon and hexagon cases. In section 4 we derive new formulae for reducing loop integrals in the singular cases where kinematic determinants vanish. In section 5 , the connection to a type of maximal cut on the pentagon is discussed.

\section{Review and definitions}

Basing our notation and analysis on $[25,26]$, a general $D$-dimensional one-loop scalar integral corresponding to the momentum routing in figure 1 is defined as

$$
\mathcal{I}_{n}^{D}=\int \frac{d^{D} \ell}{(2 \pi)^{D}} \frac{1}{\left(\ell^{2}-M_{1}^{2}\right)\left(\left(\ell-q_{2}\right)^{2}-M_{2}^{2}\right) \cdots\left(\left(\ell-q_{n}\right)^{2}-M_{n}^{2}\right)},
$$

where the momenta

$$
q_{i}=p_{1}+p_{2}+\ldots+p_{i-1}
$$

Upon integration, the denominator can be expressed as a homogeneous second-order polynomial of Feynman parameters $a_{i}$

$$
\begin{aligned}
i(-1)^{n+1}(4 \pi)^{D / 2} \mathcal{I}_{n}^{D} & =\Gamma(n-D / 2) \int_{0}^{1} d^{n} a_{i} \delta\left(1-\Sigma_{i} a_{i}\right) \frac{1}{\left[\sum_{i, j=1}^{n} a_{i} S_{i j} a_{j}\right]^{n-D / 2}} \\
& \equiv I_{n}[1] ;
\end{aligned}
$$

where the $n$-gon matrix ${ }^{3}$

$$
S_{i j}=\frac{1}{2}\left(M_{i}^{2}+M_{j}^{2}-q_{i j}^{2}\right),
$$

\footnotetext{
${ }^{3}$ In terms of graph polynomials, this is just a representation the $F$ (second Symanzik) polynomial as a bilinear map of the Feynman parameters.
} 
with $q_{i j}$ defined as

$$
q_{i j}=q_{j}-q_{i}=\sum_{i}^{j-1} p_{i}
$$

these fully characterise the integral together with the dimension $D$ and the numerator which we express in the argument of $I_{n}$ and is in general a monomial of Feynman parameters.

To avoid repeated explicit denotations of determinants, we introduce

$$
\Upsilon_{n} \equiv \operatorname{det} S
$$

and the Gram determinant, $\Delta_{n} \equiv \operatorname{det}\left[2 p_{i} \cdot p_{j}\right]$, can be expressed in terms of the $n$-gon matrix as a Cayley-Menger determinant:

$$
\Delta_{n}=2^{n-1}\left|\begin{array}{cc}
0 & -\mathbf{1}^{T} \\
\mathbf{1} & S
\end{array}\right|
$$

where 1 here represents a column of $n$ entries of value 1 .

We can derive linear relations between integrals that are useful in practical calculations by considering alternative ways of deriving the same quantities [26]. Consider the cancellation of a propagator in eq. (2.1):

$$
J_{1}=i(-1)^{n+1}(4 \pi)^{D / 2} \int \frac{d^{D} \ell}{(2 \pi)^{D}} \frac{\ell^{2}-M_{1}^{2}}{\left(\ell^{2}-M_{1}^{2}\right)\left(\left(\ell-q_{1}\right)^{2}-M_{2}^{2}\right) \cdots\left(\left(\ell-q_{n-1}\right)^{2}-M_{n}^{2}\right)} ;
$$

whence we can derive a relation by considering the evaluation of this integral in two different ways. Firstly, the obvious

$$
J_{1}=-I_{n-1}^{(1)}[1]
$$

comes from a cancellation with the propagator; the superscript (1) here means pinching the propagator between $n$ and 1. Secondly, leaving the numerator in place and Feynman parametrising leads to the identity

$$
-I_{n-1}^{(1)}[1]=(n-1-D) I_{n}^{D+2}[1]-2 \sum_{j=1}^{n} S_{1 j} I_{n}\left[a_{j}\right] .
$$

Generalising to any inverse propagator in the numerator and, where $\Upsilon_{n} \neq 0$, inverting gives

$$
I_{n}\left[a_{i}\right]=\frac{1}{2}\left[\sum_{j=1}^{n} S_{i j}^{-1} I_{n-1}^{(j)}[1]+(n-1-D) \sum_{j=1}^{n} S_{i j}^{-1} I_{n}^{D+2}[1]\right] .
$$

Summing the left-hand side using $\sum_{i=1}^{n} a_{i}=1$ gives

$$
I_{n}[1]=\frac{1}{2}\left[\sum_{j=1}^{n} c_{j} I_{n-1}^{(j)}[1]+(n-1-D) c_{0} I_{n}^{D+2}[1]\right],
$$

where we have now defined

$$
c_{i}=\sum_{j=1}^{n} S_{i j}^{-1}
$$


and

$$
\begin{aligned}
c_{0} & \equiv \sum_{j=1}^{n} c_{j} \\
& =(2)^{1-n} \frac{\Delta_{n}}{\Upsilon_{n}} .
\end{aligned}
$$

Equation (2.12) clearly diverges if $\Upsilon_{n}=0$. Introducing $L$ for the dimension of the external momenta, then the condition on the vanishing of $\Upsilon_{n}$ is

$$
n>L+2 \text {. }
$$

We explore the case where $L=4$ in section 3 , and derive the formula for the general- $L$ cases defined by equation (2.15) in section 4 .

Assuming generic parameters $\left\{M_{i}, q_{i}\right\}$ to avoid IR divergences allows us to set $D$ to be an integer, and leads to the integer version of the $n=L+1$ reduction formula

$$
I_{L+1}[1]=\frac{1}{2} \sum_{j=1}^{n} c_{j} I_{L}^{(j)}[1]
$$

This case was proved long ago [20-24], and indeed the generalisation to higher $n$ was also proved, first by Melrose [22] and very shortly afterwards by Petersson [23]. Continuation of this to the case where $D$ is not an integer is the main result of this paper.

It is worthwhile highlighting that Petersson [23] defines the $c_{i}$ in a different manner:

$$
c_{i}=\frac{1}{\left(l_{i}^{+}-q_{i-1}\right)^{2}-M_{i}}+\frac{1}{\left(l_{i}^{+}-q_{i-1}\right)^{2}-M_{i}},
$$

where $l_{i}^{ \pm}$are the two solutions to the $L$ equations

$$
\left(\ell-q_{j-1}\right)^{2}-M_{j}^{2}=0 \quad j \neq i .
$$

These can be recognised as maximal cut constraints [34] for the $L$-point integral $I_{L}^{(i)}$, although they were not considered as such at the time. This is expanded upon for the $L=4$ case and more generally to non-integer $D$ in section 5 .

\section{Four-dimensional Minkowski space}

The various kinematic coefficients $\left(c_{i}, \Upsilon_{n}, \Delta_{n}\right.$ etc...) can of course be enumerated using computer algebra systems, however we show in this section that it is in fact quite easy to derive conveniently compact expressions where $L=4$ and the internal masses are degenerate.

\subsection{Pentagon}

Our first example is the version of the shift relation (2.12) which reduces the pentagon, $I_{5}$, to boxes, $I_{4}^{(j)}$, and a dimension shifted pentagon

$$
I_{5}=\frac{1}{2}\left[\sum_{j=1}^{5} c_{j} I_{4}^{(j)}+(4-D) c_{0} I_{5}^{D+2}\right] .
$$


The Gram determinant is given by

$$
\begin{aligned}
\Delta_{5} & =\operatorname{det}_{i, j \neq 5}\left[2 p_{i} \cdot p_{j}\right] \\
& =16 \operatorname{det}_{i \neq 5}\left[p_{i \mu}\right] \operatorname{det}_{j \neq 5}\left[p_{j}^{\mu}\right] \\
& =-16 \varepsilon^{p_{1} p_{2} p_{3} p_{4}} \varepsilon_{p_{1} p_{2} p_{3} p_{4}} \\
& =\operatorname{tr}_{5}^{2}(1234) \equiv\left(\operatorname{tr}\left(\gamma_{5} \not p_{1} \not p_{2} \not p_{3} \not p_{4}\right)\right)^{2} .
\end{aligned}
$$

For simplicity considering the all-massless (i.e. propagators as well as external momenta) case, then the pentagon determinant is, written in Mandlestam $\left(s_{i j}=\left(p_{i}+p_{j}\right)^{2}\right)$ notation,

$$
\Upsilon_{5}=\left(-\frac{1}{2}\right)^{5}\left|\begin{array}{ccccc}
0 & 0 & s_{12} & s_{45} & 0 \\
0 & 0 & 0 & s_{23} & s_{51} \\
s_{12} & 0 & 0 & 0 & s_{34} \\
s_{45} & s_{23} & 0 & 0 & 0 \\
0 & s_{51} & s_{34} & 0 & 0
\end{array}\right|=-\frac{1}{16} s_{12} s_{23} s_{34} s_{45} s_{51}
$$

The $c_{i}$ 's can readily be determined by noting that, from conservation of momentum

$$
-\frac{1}{2}\left(\begin{array}{lllll}
0 & 0 & 1 & 1 & 0 \\
0 & 0 & 0 & 1 & 1 \\
1 & 0 & 0 & 0 & 1 \\
1 & 1 & 0 & 0 & 0 \\
0 & 1 & 1 & 0 & 0
\end{array}\right)\left(\begin{array}{c}
\operatorname{tr}(4512) \\
\operatorname{tr}(5123) \\
\operatorname{tr}(1234) \\
\operatorname{tr}(2345) \\
\operatorname{tr}(3451)
\end{array}\right)=\left(\begin{array}{l}
s_{23} s_{34} \\
s_{34} s_{45} \\
s_{45} s_{51} \\
s_{51} s_{12} \\
s_{12} s_{23}
\end{array}\right)
$$

and as

$$
S_{i j} c_{j}=1
$$

we can identify

$$
c_{i}=-\frac{1}{16 \Upsilon_{5}} s_{(i+1)(i+2)} s_{(i+2)(i+3)} \operatorname{tr}((i-2)(i-1) i(i+1)) .
$$

The indices take on a cyclic definition (i.e. $i+1=1$ where $i=5$ ).

\subsection{Hexagon}

At $n=6$ the reduction formula (2.12) is simplified by the fact that $\Delta_{6}=0$ for $L=4$, and thus a hexagon can be written entirely in terms of pentagons [26]

$$
I_{6}[1]=\frac{1}{2} \sum_{j=1}^{6} c_{j} I_{5}^{(j)}[1] .
$$

It is useful to have a compact value for $\Upsilon_{6}$. It was shown explicitly [35] that for the all-massless case

$$
\Upsilon_{6}=-\frac{1}{64} \operatorname{tr}_{5}^{2}(123456)
$$


This is in fact also the case for the generic external mass $\left(\left\{p_{i}^{2}=m_{i}^{2}\right\}\right)$ and equal internal mass $\left(\left\{M_{i}^{2}=M^{2}\right\}\right)$ case; this can be shown by considering the Cayley-Menger matrix $C$

$$
C=\left(\begin{array}{cc}
0 & -1 \\
1 & S
\end{array}\right)
$$

The minors of $C$ can be identified for the diagonal elements, by using the fact that they correspond pentagon Cayley-Menger determinants which, from equation (2.7), are proportional to Gram determinants. Explicitly the adjugate to the matrix $C$ can be partially filled in

$$
C^{\text {adj }}=\left(\begin{array}{ccccccc}
\Upsilon_{6} & * & * & * & * & * & * \\
* & -32 \Delta_{5}^{(1)} & * & * & * & * & * \\
* & * & -32 \Delta_{5}^{(2)} & * & * & * & * \\
* & * & * & -32 \Delta_{5}^{(3)} & * & * & * \\
* & * & * & * & -32 \Delta_{5}^{(4)} & * & * \\
* & * & * & * & * & -32 \Delta_{5}^{(5)} & * \\
* & * & * & * & * & * & -32 \Delta_{5}^{(6)}
\end{array}\right),
$$

where $\Delta_{5}^{(j)}$ is the Gram determinant of the pentagon with the $j$ th propagator pinched, and $*$ represents entries yet to be determined.

From the fact that $C$ is a symmetric $7 \times 7$ matrix of rank $6, C^{\text {adj }}$ must be a symmetric rank 1 matrix, thus expressible as the outer product of two instances of the same vector $b=\left(b_{0}, \mathbf{b}\right)^{T}$

$$
C_{i j}=b_{i} b_{j} i, j \in\{0, \ldots, 6\},
$$

where $b$ satisfies the conditions

$$
\begin{aligned}
& b_{1}^{2}=\Upsilon_{6}, \\
& b_{i}^{2}=32 \Delta_{5}^{(i-1)} \quad i \neq 0 .
\end{aligned}
$$

As

$$
C C^{\text {adj }}=\operatorname{Diag}\left(\Delta_{6}\right)=0,
$$

we can deduce from equation (2.14) that

$$
\begin{aligned}
\mathbf{1} \cdot \mathbf{b} & =\sum_{j=1}^{6} b_{j}=0, \\
\sum_{j=1}^{6} S_{i j} \cdot b_{j+1} & =-b_{0} .
\end{aligned}
$$

The latter two conditions imply that

$$
c_{i}=-\frac{b_{i}}{b_{0}} .
$$

For equal mass propagators the identity

$$
\sum_{j=1}^{6} S_{i j}(-1)^{j} \operatorname{tr}_{5}((j+1)(j+2)(j+3)(j+4))=\frac{1}{2} \operatorname{tr}_{5}(123456)
$$


can be used to deduce that

$$
\begin{aligned}
c_{i} & =(-1)^{i} \frac{2 \operatorname{tr}_{5}((i+1)(i+2)(i+3)(i+4))}{\operatorname{tr}_{5}(123456)} \\
\Rightarrow c_{i}^{2} & =4 \frac{\Delta_{5}^{(i)}}{\operatorname{tr}_{5}^{2}(123456)}
\end{aligned}
$$

and matching with equations. (3.17), (3.12) and (3.13) gives

$$
\Upsilon_{6}=-\frac{1}{64} \operatorname{tr}_{5}^{2}(123456) \text {. }
$$

This remarkably compact form of the hexagon determinant is valid for all cases where the external momenta $p_{i}$ are massive or null, but the propagators have equal mass. These expressions are practically useful for computing loop amplitudes or form factors with six or more external legs and equal internal masses, especially when paired with the analysis in the following sections. A Petersson style analysis in terms of the unparametrised Feynman integral is carried out in section 5 .

\section{Reduction formulae}

We begin this section by introducing a specific notation for the kinematic coefficients of the reduction formula (2.12) in the $L+2 \rightarrow L+1$ case

$$
\xi_{i} \equiv c_{i} \quad \text { for } L+2 \rightarrow L+1-\text { point reduction . }
$$

In this section we show that these particular variables are all that are needed for the $n \geq L+2$ reduction. In particular we show that the coefficients of reduction consist simply of a product of the $\xi \mathrm{s}$ corresponding to all possible descendant $(L+2)$-gons of the given $n$-gon. Conversely, a reduction to $(n-1)$-gons requires a choice of $(L+2)$-dimensional basis with coefficients corresponding to the $\xi$ s from a reduction of the $(L+2)$-gon complementary to said basis; this is illustrated in figure 2 below. We also explain how to deal with Feynman parameters in the numerator.

\subsection{Generalised reduction of scalar integrals}

The $L$-dimensional version of equation (3.7) is

$$
I_{L+2}=\frac{1}{2} \sum_{j=1}^{L+2} \xi_{j} I_{L+1}^{(j)},
$$

and with this notation, the reduction of a general descendant $L+2$-gon is

$$
I_{L+2}^{\left(i_{1}, i_{2}, \ldots, i_{n-L-2}\right)}=\sum_{j \in \mathcal{E}_{L+2}^{\left(i_{1}, i_{2}, \ldots i_{n-L-2}\right)}} \xi_{j}^{\left(i_{1}, i_{2}, \ldots, i_{n-L-2}\right)} I_{L+1}^{\left(i_{1}, i_{2}, \ldots, i_{n-L-2}, j\right)} ;
$$

where $\mathcal{E}_{L+2}^{\left(i_{1}, i_{2}, \ldots, i_{n-L-2}\right)}$ is the set $\{1, \ldots, n\} /\left\{i_{1}, i_{2}, \ldots i_{n-L-2}\right\}$ which indexes the descendant $(L+2)$-gon. 
It should be emphasised that there are two notation schemes for integral reduction: descendant notation indexed by the propagators shrunk from the ancestor $n$-gon, and the ascendant notation indexed by the propagators expanded. To translate between the two

$$
I^{\left(i_{1}, i_{2}, \ldots, i_{n-L-2}\right)} \equiv I^{\left[\bar{i}_{1}, \bar{i}_{2}, \ldots, \bar{i}_{L+2}\right]} \quad \text { for } \bar{i}_{k} \in \mathcal{E}_{L+2}^{\left(i_{1}, i_{2}, \ldots, i_{n-L-2}\right)},
$$

where the descendant notation is on the left and the ascendant notation is on the right.

To generalise equation (4.2) to $n>L+2$, we start by looking at the $n=L+3$ case. Equation (2.10) gives

$$
-I_{L+2}^{(i)}[1]=(L+1-D) I_{L+3}^{D+2}[1]-2 \sum_{j=1}^{n} S_{i j} I_{L+3}\left[a_{j}\right],
$$

which with some linear algebra (shown in appendix A.1) leads to

$$
I_{L+3}=\frac{1}{2} \sum_{j \in \mathcal{E}_{L+2}} \xi_{i}^{(k)} I_{L+2}^{(i)} .
$$

The manifest symmetry is broken by an explicit choice of descendant $(L+2)$-gon (the choice of pinched propagator $k$ ), however it is restored at the level of $(L+1)$-gons

$$
I_{L+3}=\frac{1}{4} \sum_{j, k=1}^{n} \xi_{k}^{(j)} \xi_{j}^{(k)} I_{L+1}^{(j, k)}
$$

The derivation generalises without any new algebraic obstruction aside from the opacity of heavily indexed notation: the reduction formula for $I_{n}$ to $I_{n-1}$ with a choice of basis corresponding to a given choice of complementary $L+2$-gon is

$$
I_{n}=\frac{1}{2} \sum_{j \in \mathcal{E}_{L+2}} \xi_{j}^{\left[k_{1}, \ldots, k_{L-2}\right]} I_{n-1}^{(j)}, \quad \text { for } n \geq L+2,
$$

where $\left\{k_{1}, \ldots, k_{n}\right\}=\{1, \ldots, n\} /\left\{i_{1}, \ldots, i_{n-L-2}\right\}$, the $i_{n}$ s being the pinched propagators from the $n$-gon to form the $(L+2)$-gon. ${ }^{4}$ For example, when $L=4$, the choice of reduction basis of $I_{n}$ corresponds to choosing the hexagon depicted in figure 2 .

The general formula for the reduction to $(L+1)$-gons is

$$
\begin{aligned}
& I_{n}=\frac{1}{2^{n-L-1}} \sum_{i_{1}, \ldots, i_{n-L-1}=1}^{n}\left[\prod_{m}^{n-L-1} \xi_{i_{m}}^{\left[\mathcal{P}, i_{m}\right]}\right] I_{L+1}^{[\mathcal{P}]}, \quad \text { for } n \geq L+2, \\
& \mathcal{P}_{i}=\left\{k_{1}, k_{2}, \ldots, k_{L+1}\right\}=\{1, \ldots, n\} /\left\{i_{1}, \ldots, i_{n-L-1}\right\} .
\end{aligned}
$$

For the $L=4$ equal-internal-mass case: ${ }^{5}$

$$
\xi_{k_{j}}^{\left[k_{1}, k_{2}, k_{3}, k_{4}, k_{5}, k_{6}\right]}=(-1)^{j} \frac{2 \operatorname{tr}_{5}\left(q_{k_{j+1} k_{j+2}} q_{k_{j+2} k_{j+3}} q_{k_{j+3} k_{j+4}} q_{k_{j+4} k_{j+5}}\right)}{\operatorname{tr}_{5}\left(q_{k_{1} k_{2}} q_{k_{2} k_{3}} q_{k_{3} k_{4}} q_{k_{4} k_{5}} q_{k_{5} k_{6}} q_{k_{6} k_{1}}\right)},
$$

with the $j s$ defined cyclically over $\{1, \ldots, 6\}$.

\footnotetext{
${ }^{4}$ This is a more compact labelling of each $(L+1)$-gon in the large- $n$ case, but less in the small- $n$ case as, for example, $I_{5}^{(1)} \equiv I_{5}^{[1,2,3,4,5]}$ for a hexagon descendant.

${ }^{5}$ It is a remarkable feature of $\operatorname{tr}_{5}$ that the expression in equation (4.10) is so broadly applicable.
} 

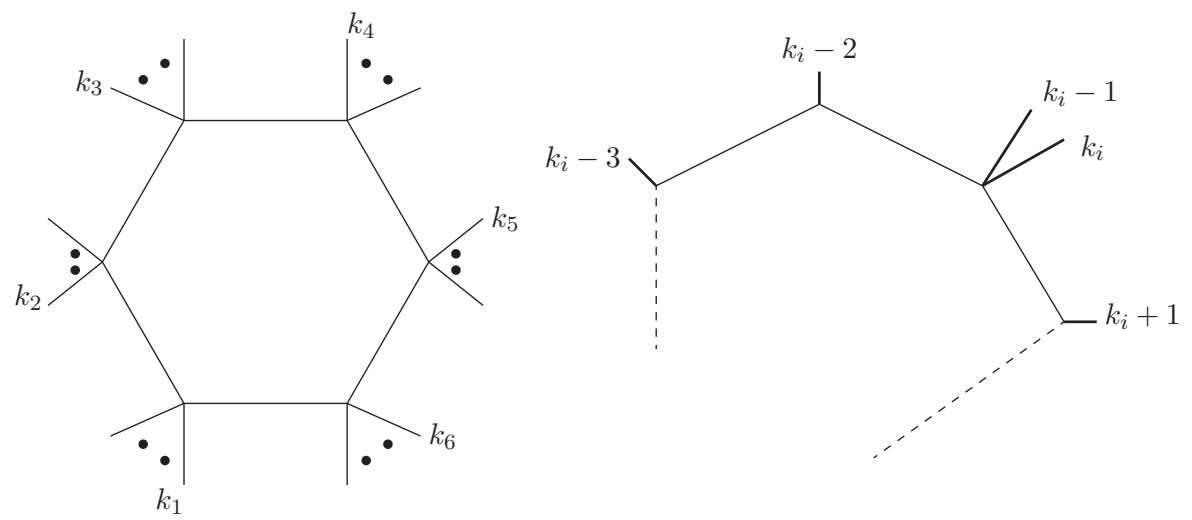

Figure 2. Left: a choice of hexagon is determined by a choice of six external legs of $I_{n}$. The coefficients are the $\xi_{i}$ s corresponding to the reduction of this hexagon to pentagons. Right: the basis of the reduction it corresponds to are the six $(n-1)$-gons which are not ancestors of this hexagon.

\subsection{Feynman parameters in the numerator}

It is sometimes necessary to reduce $n$-gons with Feynman parameters in the numerator. In [26], a formula is derived for the reduction of an $n$-point integral with a pair of Feynman parameters in the numerator. We repeat the derivation here for completeness.

We begin with a method analagous to used in section 2

$$
\left.J_{n ; i} \equiv \Gamma(n-1-D / 2) \int_{0}^{1} \cdots \int_{0}^{1-a_{1}-a_{2}-\ldots \hat{a}_{i} \ldots-a_{n-1}} d a_{n} \frac{d}{d a_{n}}\left[\frac{a_{k}}{\left(\mathbf{a}^{\mathrm{T}} S \mathbf{a}\right)^{n+1-D / 2}}\right]\right|_{a_{i}=1-a_{1} \ldots-a_{n}} ;
$$

the $\hat{a}_{i}$ signifies the omission of $a_{i}$; applying the derivative gives

$$
J_{n ; i, k}=\left(\delta_{n k}-\delta_{i k}\right) I_{n}^{D+2}-2 \sum_{j=1}^{n}\left(S_{n j}-S_{i j}\right) I_{n}\left[a_{j} a_{k}\right] .
$$

Alternatively, integration gives

$$
J_{n ; i}=I_{n-1}^{(i)}\left[a_{k}\right]-I_{n-1}^{(n)}\left[a_{k}\right],
$$

where $I^{(k)}\left[a_{k}\right]=0$.

Now combining (4.12) and (4.13) allows us to define

$$
\Phi_{k} \equiv \delta_{i k} I_{n}^{D+2}-2 \sum_{j=1} S_{i j} I_{n}\left[a_{j} a_{k}\right]+I_{n-1}^{(i)}\left[a_{k}\right]
$$

which is independent of the choice of index $i$ on the right-hand side.

We assume $\Upsilon_{n} \neq 0$ and $\Delta_{n} \neq 0$; using the trick

$$
\sum_{j} S_{i j}^{-1} \Phi_{k}=S_{i k}^{-1} I_{n}^{D+2}-2 I_{n}\left[a_{i} a_{k}\right]+S_{i j}^{-1} I_{n-1}^{(j)}\left[a_{k}\right],
$$

where, also

$$
\sum_{j} S_{i j}^{-1} \Phi_{k}=c_{i} \Phi_{k}
$$


and using the fact that $\sum_{j} S_{l j} c_{j}=1$, then

$$
c_{0} \Phi_{k}=c_{0} I_{n}^{D+2}-2 I_{n}\left[a_{k}\right]+c_{j} I_{n-1}^{(j)}\left[a_{k}\right] .
$$

Combining with equations. (4.15) and (2.12) gives

$$
\begin{aligned}
I_{n}\left[a_{i} a_{k}\right]= & \frac{1}{2}\left[S_{i k}^{-1}+(n-2-D) \frac{c_{i} c_{k}}{c_{0}}\right] I_{n}^{D+2} \\
& +\frac{1}{4}(n-2-D)\left[c_{k} S_{i j}^{-1}+c_{i} S_{k j}^{-1}-\frac{c_{i} c_{k} c_{j}}{c_{0}}\right] I_{n-1}^{(j) ; D+2}+\frac{1}{4} S_{i j}^{-1} S_{k l}^{-1} I_{n-2}^{(j, l)},
\end{aligned}
$$

where we implicitly sum over $j$ consistent with summation convention. Note that equation (4.18) applies for all cases where $n \leq L+1$; a good consistency check can be carried out by confirming that summing equation (4.18), $\sum_{j=1}^{n} I_{n}\left[a_{j} a_{i}\right]=I_{n}\left[a_{i}\right]$, reduces to equation (2.11).

For $S_{i k} \neq 0$ we identify

$$
I_{n}\left[a_{i} a_{k}\right]=-\frac{1}{2} \frac{\partial}{\partial S_{i k}} I_{n}^{D+2}[1],
$$

while for a $S_{i k}=0$, these can be related to a non-zero entry through $\Phi_{k}$ equivalence.

The $n=L+2\left(\Delta_{n}=c_{0}=0\right)$ case can also be derived,

$$
I_{L+2}\left[a_{i} a_{k}\right]=-\frac{1}{2} \frac{\partial}{\partial S_{i k}}\left(I_{L+2}^{D+2}\right)=\frac{1}{2}\left[\sum_{j=1}^{L+2} \xi_{j} I_{L+1}^{(j)}\left[a_{i} a_{k}\right]-\frac{1}{2} \sum_{j=1}^{L+2} \frac{\partial \xi_{j}}{\partial S_{i k}} I_{L+1}^{(j)}[1]\right] ;
$$

and making the observation that

$$
\begin{aligned}
(S \xi)_{l} & =1 \\
\Rightarrow\left(\frac{\partial S \xi}{\partial S_{i k}}\right)_{l} & =0 \\
\Rightarrow \frac{\partial \xi_{l}}{\partial S_{i k}} & =-\left(S_{i l}^{-1} \xi_{k}+S_{k l}^{-1} \xi_{i}\right)
\end{aligned}
$$

gives the generic formula for a monomial of Feynman parameters in terms of lower-point amplitude.

The reduction of any $n$-point integral for $n>L+2$ follows by replacing the reduction in equation (4.20) by the relevant choice of reduction formulae; the matrix $S$ used in the simplification of the derivative in equation (4.22) needs to be replaced by the appropriate $L+2$-gon $S_{i j}$ matrix for each $\xi$, as described in the previous section.

Note that combining equation (4.18) with equation (4.19) generates the partial differential equations for $I_{n}^{D+2}$ in terms of the variables $S_{i k}$.

We also note here that the analysis generalises to terms with a greater number of Feynman parameters in the numerator, $N$, by generalising $\Phi_{k} \rightarrow \Phi_{\mathbf{k}} \equiv \Phi_{k_{1} k_{2} \ldots k_{N}}$

$$
\Phi_{\mathbf{k}} \equiv \sum_{j=1}^{N} \delta_{i k_{l}} I_{n}^{D+2}\left[a_{k_{1}} a_{k_{2}} \cdots \hat{a}_{k_{l}} \cdots a_{k_{N}}\right]-2 S_{i j} I_{n}\left[a_{j} \prod_{l=1}^{N} a_{k_{l}}\right]+I_{n-1}^{(i)}\left[\prod_{l=1}^{N} a_{k_{l}}\right] .
$$

We leave such derivations to the reader. 


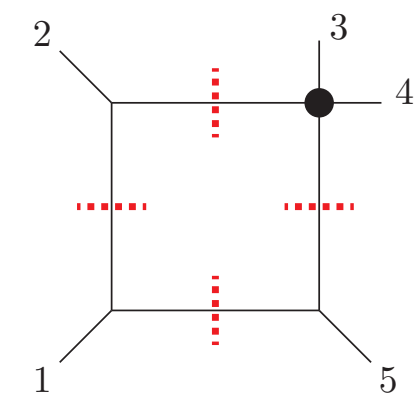

Figure 3. The quadruple cut can be solved and the solutions plugged into the pinched propagator to give $c_{4}$.

\section{Maximal cuts}

The Petersson formula (2.17) implies there is another way of finding $\mathbf{c}$ by solving the on-shell conditions of the one-mass boxes of the kind depicted in figure 3 .

Without loss of generality, we consider $c_{4}$; the on-shell conditions read

$$
\begin{aligned}
\ell^{2} & =0, \\
\left(\ell-p_{1}\right)^{2} & =0, \\
\left(\ell-p_{1}-p_{2}\right)^{2} & =0, \\
\left(\ell+p_{5}\right)^{2} & =0 ;
\end{aligned}
$$

their two solutions lie in complex momentum space, expressed in the spinor-helicity formalism as

$$
\begin{aligned}
\ell_{a \dot{b}}=l_{a \dot{b}}^{+} & =\frac{[12]}{[52]} \lambda_{1} \bar{\lambda}_{5}, \\
l_{a \dot{b}}^{-} & =\frac{\langle 12\rangle}{\langle 52\rangle} \lambda_{5} \bar{\lambda}_{1} .
\end{aligned}
$$

Applying these solutions into the pinched propagator:

$$
\begin{aligned}
& \left(l^{+}-q_{3}\right)^{2}=\frac{[2|34| 5]}{[52]}, \\
& \left(l^{-}-q_{3}\right)^{2}=\frac{\langle 2|34| 5\rangle}{\langle 52\rangle} .
\end{aligned}
$$

so that, using the definition (2.17),

$$
\begin{aligned}
c_{4} & =\frac{[52]}{[2|34| 5]}+\frac{\langle 52\rangle}{\langle 2|34| 5\rangle} \\
& =\frac{\operatorname{tr}(2345)}{s_{23} s_{34} s_{45}}
\end{aligned}
$$




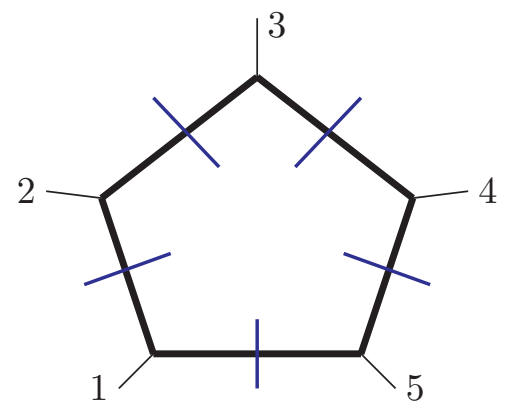

Figure 4. The pentagon $D$-dimensional pole corresponds to the unique solution $l_{0}$ to the cut constraints (5.10).

which matches equation (3.6), showing consistency between the "Feynman-parametric", $S_{i j}$-matrix-based Melrose picture [22] and the unparametrised cut-constraint based Petersson analysis [23].

\subsection{The $D$-dimensional pole}

Here we look at the singular behaviour missed by the four-dimensional cut constraints. Again specifying to the $L-4$ case, the $4-2 \epsilon$ pentagon dimension-shift identity (2.12) is

$$
I_{5}=\frac{1}{2}\left[\sum_{j=1}^{n} c_{j} I_{4}^{(j)}+\epsilon c_{0} I_{5}^{6-2 \epsilon}\right] .
$$

This equation has interesting properties when one considers the maximal cut of the pentagon. Cut conditions for the equal internal mass pentagon depicted in figure 4 are

$$
\begin{aligned}
\ell^{2}-M^{2}=\ell^{[4] 2}+\ell^{[-2 \epsilon] 2}-M^{2}=l^{2}-\mu^{2} & =0, \\
\left(l-p_{1}\right)^{2}-\mu^{2} & =0, \\
\left(l-p_{1}-p_{2}\right)^{2}-\mu^{2} & =0, \\
\left(l+p_{5}+p_{4}\right)^{2}-\mu^{2} & =0, \\
\left(l+p_{5}\right)^{2}-\mu^{2} & =0 ;
\end{aligned}
$$

where we have defined $\mu^{2}=M^{2}-\ell^{[-2 \epsilon] 2}$. The unique solution to the conditions (5.10) is

$$
\begin{aligned}
& l_{0}^{\mu}=\frac{\operatorname{tr}_{5}\left(\gamma^{\mu} 12345\right)}{2 \operatorname{tr}_{5}(2345)} \\
& \mu_{0}^{2}=l^{2}=\frac{16 \Upsilon}{\Delta_{5}}=\frac{1}{c_{0}} .
\end{aligned}
$$

where in the spinor helicity formalism, if e.g. the external momenta 5 and 1 are massless $\left(m_{1}^{2}=m_{2}^{2}=0\right)$,

$$
\operatorname{tr}_{5}\left(\sigma^{\dot{a} b} 12345\right)=\lambda_{1}^{\dot{a}}[1|234| 5\rangle \bar{\lambda}_{5}^{b}-\lambda_{5}^{\dot{a}}[5|432| 1\rangle \bar{\lambda}_{1}^{b}
$$

which can comfortably be generalised to the massive case over a given basis using the various schemes available [36-39]. 
Considering the integral explicitly and re-introducing the normalisation of equation $(2.1)$

$$
(2 \pi)^{4-2 \epsilon} \mathcal{I}_{5}=\int \frac{d^{4-2 \epsilon} \ell}{\left(\ell^{2}-M^{2}\right)\left(\left(\ell-q_{1}\right)^{2}-M^{2}\right)\left(\left(\ell-q_{2}\right)^{2}-M^{2}\right)\left(\left(\ell-q_{3}\right)^{2}-M^{2}\right)\left(\left(\ell-q_{4}\right)^{2}-M^{2}\right)},
$$

we follow the standard procedure of separating the integral measure:

$$
d^{4-2 \epsilon} \ell=-\frac{1}{2} \Omega_{S_{-2 \epsilon}} \frac{d^{4} l d \mu^{2}}{\left(\mu^{2}\right)^{1+\epsilon}}
$$

where $\Omega_{S_{-2 \epsilon}}$ is the unit surface area of the sphere, $S_{-2 \epsilon}$, as is normally extracted from the loop integration step. Upon shifting the $\mu^{2}$ variable such that it matches the definition from the conditions (5.10), this gives the integral

$$
\begin{aligned}
= & -\frac{1}{2} \Omega_{S_{-2 \epsilon}} \int_{M^{2}}^{\infty} \frac{d \mu^{2}}{\left(\mu^{2}-M^{2}\right)^{1+\epsilon}} \\
& \times \int \frac{d^{4} l}{\left(l^{2}-\mu^{2}\right)\left(\left(l-q_{1}\right)^{2}-\mu^{2}\right)\left(\left(l-q_{2}\right)^{2}-\mu^{2}\right)\left(\left(l-q_{3}\right)^{2}-\mu^{2}\right)\left(\left(l-q_{4}\right)^{2}-\mu^{2}\right)} .
\end{aligned}
$$

The residues of the integrand can be captured changing the $\mu^{2}$ integration region to a large enough contour on the complex plane, and taking the limit $\epsilon \rightarrow 0$; we can see that where the "four-dimensional" residue is simply manifestly captured by the pole at

$$
\mu^{2}=M^{2}
$$

and need only be combined with four other conditions (for example the box cut conditions (5.4)).

The $D$-dimensional pole, on the other hand, is buried in the solution to the on-shell conditions (5.10); it lies at

$$
\mu^{2}=\frac{1}{c_{0}}
$$

It is not a coincidence that this is the inverse of the coefficient of the shifted integral in equation (2.12). as indicated by Schnetz for the Petersson formula in the integer dimension case [40], one can view the cut version of equation (5.9) as being analagous to a sum over residues; it can be seen as a "freezing out" of propagators into rational coefficients.

This analysis can be carried forward to the $n$-gon reduction formula (4.9); this is straightforward as we observe that in the hexagon case

$$
I_{6}=\frac{1}{2} \sum_{j=1}^{6} \xi_{j} I_{5}^{(j)}
$$

taking the solution (5.11) for e.g. $I_{5}^{(6)}$ gives

$$
\left(\ell+p_{6}\right)=2 l_{0} \cdot p_{6}+p_{6}^{2}=\frac{\operatorname{tr}_{5}(612345)}{\operatorname{tr}_{5}(1234)}=\frac{2}{\xi_{6}} .
$$


Consistent with the Petersson formula for a hexagon, the coefficient of the descendant box solutions satisfy the equations

$$
\frac{1}{2}\left(\xi_{i} c_{j}^{(i)}+\xi_{j} c_{i}^{(j)}\right)=\frac{1}{Q_{i}\left(l^{+}\right) Q_{j}\left(l^{+}\right)}+\frac{1}{Q_{i}\left(l^{-}\right) Q_{j}\left(l^{-}\right)},
$$

where $Q_{i}\left(l^{ \pm}\right)$is the inverse "frozen out" $i$ th propagator with the null cut solutions of the other propagators $l^{ \pm}$inserted. Note that the Petersson form on the right-hand side of equation (5.21) does not factor in such a simple manner, but is averaged over the all positive and all negative products. In this sense the unique solution to the $D$-dimensional constraint is simpler as it avoids the need to average:

$$
\prod_{m}^{n-L-1} \xi_{i_{m}}^{\left[\mathcal{P}, i_{m}\right]}=\prod_{m}^{n-L-1} \frac{2^{n-L-2}}{Q\left(\ell_{I_{m}}\right)} .
$$

\section{Concluding remarks}

The formulae presented in this paper for the reduction of integrals should greatly simplify high-multiplicity amplitude computations. As well as being useful for reducing highmultiplicity integrals, the reduction formulae (4.9) also presents a very simple interpretation in terms of "freezing out" of propagators.

This integration with unitarity cuts leaves open the prospect of computing all-epsilon, all-multiplicity closed forms for scattering amplitudes, which as well as being a step forwards methodically, could also further more general analytic understanding of scattering amplitudes. The remarkable simplicity of the solution to the pentagon equal-mass conditions (5.10) opens up the prospect of a "generalised unitarity" approach to $D$-dimensional unitarity, avoiding the need for integrand reconstruction.

One could pose the question as to whether the "freezing out" can be extended to simplify multi-loop relations where reductions and relations between integrals are more involved.

We leave these questions to future work.

\section{Acknowledgments}

I would like to thank Ruth Britto, Riccardo Gonzo, Martijn Hidding, Andrea Orta and Johann Usovitsch for countless useful conversations during the gestation of this work. Particular thanks to Anne Spiering for helpful comments on a draft of this manuscript. Thank you also to Ruth Britto for looking over the paper.

I would also like to thank the Galileo Galilei Institute (GGI) for hosting me for the "Amplitudes in the LHC era" workshop where some of the initial parts of this work developed.

Diagrams were drawn using Axodraw.

This work was supported by the ERC "CutLoops" consolidator grant number 647356 . 


\section{A Explicit derivations}

\section{A.1 $n=L+3$ reduction}

Beginning with equation (4.5), we have

$$
-I_{L+2}^{(i)}[1]=(L+1-D) I_{L+3}^{D+2}[1]-2 \sum_{j=1}^{n} S_{i j} I_{L+3}\left[a_{j}\right],
$$

and then multiplying by $\xi_{i}^{(k)}$ and summing

$$
\begin{aligned}
\frac{1}{2} \sum_{i \in \mathcal{E}_{L+2}} \xi_{i}^{(k)} I_{L+2}^{(i)}[1] & =\sum_{i \in \mathcal{E}_{L+2}} \xi_{i}^{(k)} \sum_{j=1}^{n} S_{i j} I_{L+3}\left[a_{j}\right] \\
& =\sum_{i \in \mathcal{E}_{L+2}} \xi_{i}^{(k)} \sum_{j \in \mathcal{E}_{L+2}}^{n} S_{i j} I_{L+3}\left[a_{j}\right]+\sum_{i \in \mathcal{E}_{L+2}} \xi_{i}^{(k)} S_{i k} I_{L+3}\left[a_{k}\right]
\end{aligned}
$$

which, using the identity

$$
\sum_{i \in \mathcal{E}} S_{i j} \xi_{i}^{(k)}=1
$$

and $\sum_{j=1}^{n} a_{j}=1$ gives

$$
\frac{1}{2} \sum_{j \in \mathcal{E}_{L+2}} \xi_{i}^{(k)} I_{L+2}^{(i)}[1]=I_{L+3}[1]+\left(\sum_{i \in \mathcal{E}_{L+2}} \xi_{i}^{(k)} S_{i k}-1\right) I_{L+3}\left[a_{k}\right] .
$$

Recalling equation (2.14) (and defining $\xi_{k}^{(k)}=0$ )

$$
\sum_{i=1}^{L+3} \xi_{i}^{(k)}=2^{L+1} \frac{\Delta_{L+2}^{(k)}}{\Upsilon_{L+2}^{(k)}}=0
$$

and, as $\Upsilon_{L+3}=0$,

$$
S_{i k}=S_{i(k+1)}+\text { column operations },
$$

then we deduce that

$$
\sum_{i \in \mathcal{E}_{L+2}} \xi_{i}^{(k)} S_{i k}-1=0
$$

Thus

$$
I_{L+3}=\frac{1}{2} \sum_{j \in \mathcal{E}_{L+2}} \xi_{i}^{(k)} I_{L+2}^{(i)}
$$

which is equation (4.6).

The reduction formula (4.2) can now be applied to each $(L+2)$-gon to give

$$
I_{L+3}=\frac{1}{4} \sum_{j, k=1}^{n} \xi_{k}^{(j)} \xi_{j}^{(k)} I_{L+1}^{(j, k)},
$$

which is equation (4.7). 
Open Access. This article is distributed under the terms of the Creative Commons Attribution License (CC-BY 4.0), which permits any use, distribution and reproduction in any medium, provided the original author(s) and source are credited.

\section{References}

[1] S. Badger, G. Mogull, A. Ochirov and D. O'Connell, A complete two-loop, five-gluon helicity amplitude in Yang-Mills theory, JHEP 10 (2015) 064 [arXiv:1507.08797] [INSPIRE].

[2] S. Badger, C. Brønnum-Hansen, H.B. Hartanto and T. Peraro, Analytic helicity amplitudes for two-loop five-gluon scattering: the single-minus case, JHEP 01 (2019) 186 [arXiv: 1811.11699] [INSPIRE].

[3] S. Abreu, J. Dormans, F. Febres Cordero, H. Ita, B. Page and V. Sotnikov, Analytic form of the planar two-loop five-parton scattering amplitudes in QCD, JHEP 05 (2019) 084 [arXiv: 1904.00945] [INSPIRE].

[4] S. Badger et al., Analytic form of the full two-loop five-gluon all-plus helicity amplitude, Phys. Rev. Lett. 123 (2019) 071601 [arXiv:1905.03733] [INSPIRE].

[5] D.C. Dunbar, J.H. Godwin, W.B. Perkins and J.M.W. Strong, Color dressed unitarity and recursion for Yang-Mills two-loop all-plus amplitudes, Phys. Rev. D 101 (2020) 016009 [arXiv: 1911.06547] [INSPIRE].

[6] S. Caron-Huot, L.J. Dixon, F. Dulat, M. Von Hippel, A.J. McLeod and G. Papathanasiou, The cosmic Galois group and extended Steinmann relations for planar $N=4 S Y M$ amplitudes, JHEP 09 (2019) 061 [arXiv: 1906.07116] [INSPIRE].

[7] Z. Bern, L.J. Dixon, D.C. Dunbar and D.A. Kosower, Fusing gauge theory tree amplitudes into loop amplitudes, Nucl. Phys. B 435 (1995) 59 [hep-ph/9409265] [InSPIRE].

[8] G. Mahlon, Multi-gluon helicity amplitudes involving a quark loop, Phys. Rev. D 49 (1994) 4438 [hep-ph/9312276] [INSPIRE].

[9] G. Mahlon, Use of recursion relations to compute one loop helicity amplitudes, in $4^{\text {th }}$ international conference on physics beyond the Standard Model, (1994), pg. 475 [hep-ph/9412350] [INSPIRE].

[10] D.C. Dunbar, W.B. Perkins and J.M.W. Strong, n-point QCD two-loop amplitude, Phys. Rev. D 101 (2020) 076001 [arXiv:2001.11347] [InSPIRE].

[11] C. Anastasiou, Z. Bern, L.J. Dixon and D.A. Kosower, Planar amplitudes in maximally supersymmetric Yang-Mills theory, Phys. Rev. Lett. 91 (2003) 251602 [hep-th/0309040] [INSPIRE].

[12] Z. Bern, L.J. Dixon and V.A. Smirnov, Iteration of planar amplitudes in maximally supersymmetric Yang-Mills theory at three loops and beyond, Phys. Rev. D 72 (2005) 085001 [hep-th/0505205] [INSPIRE].

[13] T. Gehrmann, J.M. Henn and N.A. Lo Presti, Analytic form of the two-loop planar five-gluon all-plus-helicity amplitude in QCD, Phys. Rev. Lett. 116 (2016) 062001 [Erratum ibid. 116 (2016) 189903] [arXiv: 1511.05409] [INSPIRE].

[14] D.C. Dunbar and W.B. Perkins, Two-loop five-point all plus helicity Yang-Mills amplitude, Phys. Rev. D 93 (2016) 085029 [arXiv:1603.07514] [INSPIRE]. 
[15] D.C. Dunbar, J.H. Godwin, G.R. Jehu and W.B. Perkins, Analytic all-plus-helicity gluon amplitudes in QCD, Phys. Rev. D 96 (2017) 116013 [arXiv:1710.10071] [INSPIRE].

[16] J. Henn, B. Power and S. Zoia, Conformal invariance of the one-loop all-plus helicity scattering amplitudes, JHEP 02 (2020) 019 [arXiv:1911.12142] [INSPIRE].

[17] S. Catani, The singular behavior of QCD amplitudes at two loop order, Phys. Lett. B 427 (1998) 161 [hep-ph/9802439] [INSPIRE].

[18] Z. Bern, L.J. Dixon, D.C. Dunbar and D.A. Kosower, One loop selfdual and $N=4$ super Yang-Mills, Phys. Lett. B 394 (1997) 105 [hep-th/9611127] [INSPIRE].

[19] R. Britto, G.R. Jehu and A. Orta, The dimension-shift conjecture for one-loop amplitudes, arXiv: 2011.13821 [INSPIRE].

[20] L.M. Brown, Analytic properties of n-point loops in perturbation theory, Nuovo Cim. 22 (1961) 178 [INSPIRE].

[21] F.R. Halpern, Reduction formula for the five-point function, Phys. Rev. Lett. 10 (1963) 310 [INSPIRE].

[22] D.B. Melrose, Reduction of Feynman diagrams, Nuovo Cim. 40 (1965) 181 [INSPIRE].

[23] B. Petersson, Reduction of a one-loop Feynman diagram with $n$ vertices in $m$-dimensional Lorentz space, J. Math. Phys. 6 (1965) 1955 [inSPIRE].

[24] W.L. van Neerven and J.A.M. Vermaseren, Large loop integrals, Phys. Lett. B 137 (1984) 241 [INSPIRE].

[25] Z. Bern, L.J. Dixon and D.A. Kosower, Dimensionally regulated one loop integrals, Phys. Lett. B 302 (1993) 299 [Erratum ibid. 318 (1993) 649] [hep-ph/9212308] [INSPIRE].

[26] Z. Bern, L.J. Dixon and D.A. Kosower, Dimensionally regulated pentagon integrals, Nucl. Phys. B 412 (1994) 751 [hep-ph/9306240] [INSPIRE].

[27] O.V. Tarasov, Connection between Feynman integrals having different values of the space-time dimension, Phys. Rev. D 54 (1996) 6479 [hep-th/9606018] [INSPIRE].

[28] R.N. Lee, Space-time dimensionality $D$ as complex variable: calculating loop integrals using dimensional recurrence relation and analytical properties with respect to D, Nucl. Phys. B 830 (2010) 474 [arXiv:0911.0252] [INSPIRE].

[29] R.N. Lee, A.V. Smirnov and V.A. Smirnov, Dimensional recurrence relations: an easy way to evaluate higher orders of expansion in $\epsilon$, Nucl. Phys. B Proc. Suppl. 205-206 (2010) 308 [arXiv: 1005.0362] [INSPIRE].

[30] R.N. Lee and V.A. Smirnov, The dimensional recurrence and analyticity method for multicomponent master integrals: using unitarity cuts to construct homogeneous solutions, JHEP 12 (2012) 104 [arXiv: 1209.0339] [INSPIRE].

[31] V.V. Bytev, M.Y. Kalmykov and S.-O. Moch, HYPERgeometric functions DIfferential REduction (HYPERDIRE): Mathematica based packages for differential reduction of generalized hypergeometric functions. $F_{D}$ and $F_{S}$ Horn-type hypergeometric functions of three variables, Comput. Phys. Commun. 185 (2014) 3041 [arXiv:1312.5777] [InSPIRE].

[32] Z. Bern and D.A. Kosower, The computation of loop amplitudes in gauge theories, Nucl. Phys. B 379 (1992) 451 [INSPIRE].

[33] T. Binoth, J.P. Guillet and G. Heinrich, Reduction formalism for dimensionally regulated one loop $N$ point integrals, Nucl. Phys. B 572 (2000) 361 [hep-ph/9911342] [INSPIRE]. 
[34] R. Britto, F. Cachazo and B. Feng, Generalized unitarity and one-loop amplitudes in $N=4$ super-Yang-Mills, Nucl. Phys. B 725 (2005) 275 [hep-th/0412103] [INSPIRE].

[35] T. Binoth, J.P. Guillet, G. Heinrich, E. Pilon and C. Schubert, An algebraic/numerical formalism for one-loop multi-leg amplitudes, JHEP 10 (2005) 015 [hep-ph/0504267] [INSPIRE].

[36] S. Dittmaier, Weyl-van der Waerden formalism for helicity amplitudes of massive particles, Phys. Rev. D 59 (1998) 016007 [hep-ph/9805445] [INSPIRE].

[37] C. Schwinn and S. Weinzierl, Scalar diagrammatic rules for Born amplitudes in QCD, JHEP 05 (2005) 006 [hep-th/0503015] [INSPIRE].

[38] C. Cheung and D. O'Connell, Amplitudes and spinor-helicity in six dimensions, JHEP 07 (2009) 075 [arXiv: 0902.0981] [INSPIRE].

[39] N. Arkani-Hamed, T.-C. Huang and Y.-T. Huang, Scattering amplitudes for all masses and spins, arXiv:1709.04891 [INSPIRE].

[40] O. Schnetz, The geometry of one-loop amplitudes, arXiv:1010.5334 [INSPIRE]. 